duit conformément aux dispositions de l'art. a du présent décret ;

Le nom ou la raison sociale du fabricant;

La date de fabrication (millésime de l'année) dans les mêmes conditions que celles fixées par le paragraphe 4 de l'article $7 \mathrm{du}$ présent décret;

Le poids net de la marchandise exprimé en kilogs;

Les indications relatives à la dénomination du produit et au poids net doivent être présentées en caractères de dimensions au moins égales à celles des caractères les plus grands figurant sur l'emballage et de même apparence typogra. phique.

Les modifications proposées permettraient d'établir une réglementation applicable en période normale. Il est bien évident qu'en période de crise, s'il était avéré que les minima fixés par la régle. mentation normale sont trop élevés, un règlement momentané et provisoire pourrait les modifier dans les limites exigées par l'intérêt national. On en serait quitte pour revenir à la réglementation normale dès que les circonstances seraient redevenues favorables.

\title{
MISE EN ÉVIDENCE ET ISOLEMENT DES ENDUITS PROTECTEURS DE L'ALUMINIUM
}

$$
\text { par }
$$

\section{H. COUTURIER}

Depuis le début de la guerre l'aluminium laminé a progressivement remplacé l'étain dans la plupart de ses applications, notamment dans l'emballage des denrées alimentaires. Mais sa résistance à certains agents chimiques est moins grande, surtout lorsqu'il s'agit de contacts prolongés. C'est pourquoi l'on recouvre souvent la surface métallique trop altérable d'un vernis protecteur étendu en couche très mince et formant une sorte de film de quelques centièmes de millimètres d'épaisseur. 
Ce film doit être continu, c'est-à-dire sans la moindre perforation, homogène, d'épaisseur uniforme, plastique à froid et légèrement adhésif à chaud. Il doit, en outre, être inattaquable par les substances avec lesquelles il entrera en contact, inodore, sans saveur et sans toxicité. On comprendra que le fabricant, comme l'usager, aient à s'assurer souvent que ces conditions sont remplies. Pour cela, il faut isoler l'enduit de son support et l'obtenir, sous forme d'une mince pellicule dont l'examen physique et chimique devient alors possible.

La méthode courante consiste à plonger la feuille d'aluminium dans un bain d'eau régale. Le métal est rapidement dissous et la pellicule d'enduit se trouve ainsi libérée. Une telle façon de faire n'est cependant pas sans présenter de graves défauts :

$1^{0}$ Le film, qui est résistant aux acides faibles, ne l'est pas toujours aux acides forts; ses propriétés risquent done de ne plus être les mêmes avant et après traitement;

$2^{\circ}$ Le film doit être manipulé dans le bain au moyen de spatules ou de baguettes de verre : manœuvre difficile qui entraîne souvent des déchirures ou des éraillures;

$3^{\circ}$ Les projections d'eau régale sont dangereuses, et plus encore les vapeurs émises, qui peuvent occasionner de graves lésions pulmonaires, soit qu'on en respire souvent de petites quantités, soit qu'accidentellement on en inhale en une seule fois une dose massive.

C'est dans le but de supprimer ces inconvénients que nous proposons d'utiliser le puissant pouvoir de corrosion par électrolyse et par oxydation qu'ont, vis-à-vis de l'aluminium, le mercure et ses sels. L'aluminium mordancé, attaqué par le mercure ne résiste plus même à des acides faibles, même à l'oxygène de l'air.

Deux solutions sont nécessaires : l'une de bichlorure de mercure (sublimé corrosif) à $1 \%$ dans l'eau, l'autre d'acide phosphorique (ou à défaut d'acide chlorhydrique) à $10 \%$ dans l'eau. L'une et l'autre n'émettent aucune vapeur délétère. Elles sont pratiquement sans danger pour la peau et pour les vêtements, et ne présentent de toxicité que si on les boit. En langage de laboratoire, on peut les qualifier d'inoffensives.

Le mode opératoire est simple : dans une cuvette photographique contenant la solution de sublimé, on plonge la feuille d'aluminium, face vernie en dessous, face non protégée en dessus, en remuant la cuvette pour agiter le liquide. Après une ou deux minutes, le métal est devenu blanc-grisâtre mat. On vide la solution mercurique (qui pourra servir pour d'autres opérations, jusqu'à épuisement), on rince sommairement à l'eau et l'on verse la solu- 
tion acide. Bientôt de petites bulles d'hydrogène montrent que l'attaque est commencée. En dix minutes environ, si on a eu soin de remuer la cuvette, la totalité de l'aluminium est dissoute. On peut alors rincer la pellicule, la prendre avec les doigts, l'essorer entre deux feuilles de papier buvard, la sécher et l'examiner à loisir.

On pourrait remplacer l'acide phosphorique, ou l'acide chlorhydrique par d'autres acides ; mais ce sont ces deux là, l'acide phosphorique surtout, qui donnent les résultats les plus rapides. On pourrait aussi n'employer qu'un seul bain contenant à la fois le mercure et l'acide ; mais ce bain se conserve mal, et nous préférons la méthode en deux temps.

Bien entendu, s'il s'agit d'aluminium protégé sur les deux faces, cas fréquent, il faut auparavant mettre le métal à nu sur la face opposée d̀ celle où se trouve le film à isoler. Il suffit de frotter la feuille, étalée sur une plaque de verre, au moyen d'un tampon d'ouate imbibé d'un solvant approprié. Un mélange à parties égales d'alcool et d'acétone convient presque toujours ; plus rarement, il faudra avoir recours à l'éther acétique, au trichloréthylène, au furfurol, etc.

Ce procédé d'isolement est à peu de chose près aussi rapide que le procédé a l'eau régale. Il présente le triple avantage de ne pas altérer la structure du film, de rendre les manipulations faciles et de permettre de travailler à l'abri de projections ou d'émanations. dangereuses

Lorsqu'on veut non plus isoler l'enduit protecteur, mais seulement s'assurer de sa présence, l'emploi de sels de mercure sera beaucoup plus pratique que celui d'un acide et donnera des indications bien plus précises. Une solution à $5 \%$ de bichlorure de mercure dans l'eau constituera un réactif d'une puissance extraordinaire : sous son action, une feuille d'ảluminium non protégée par un enduit sera "dévorée" par oxydation en moins d'une minute, avee un échauffement tel qu'on ne peut pas la tenir dans la main. Si l'enduit est de bonne qualité, l'application du réactif ne produira aucun effet. Si l'enduit est cassé ou perforé, les défauts seront le point de départ d'une attaque très nette. Mais cette dernière méthode, basée sur l'emploi d'une solution relativement concentrée d'un sel de mercure, que l'on étend à l'aide d'un tampon d'ouate et qui sèche rapidement en agissant par oxydation, doit être réservée au simple contrôle de la présence ou de l'absence d'un enduit protecteur. L'élévation de température qui résulte de l'oxydation altère en effet le film. Aussi, toutes les fois que l'on veut isoler l'enduit protecteur en vue d'un examen chimique ou physique, c'est à la corrosion électrolytique qu'il faut avoir recours, telle qu'elle est déerite dans la première partie de ce travail. 